\title{
The structure of the circumnuclear absorber in NGC 4261 by multi-frequency phase-referencing observation
}

Takafumi Haga ${ }^{* * 1}$ Akhiro Doi, ${ }^{2}$ Yasuhiro Murata, ${ }^{2}$ Hiroshi Sudo, ${ }^{3}$ Seiji Kameno, ${ }^{4}$ and Kazuhiro $\mathrm{Hada}^{5}$

${ }^{1}$ Department of Space and Astronautical Science, The Graduate University for Advanced Studies (SOKENDAI)

3-1-1 Yoshinodai, Chuo-ku, Sagamihara, Kanagawa 252-5210, Japan

${ }^{2}$ Institute of Space and Astronautical Science, Japan Aerospace Exploration Agency

3-1-1 Yoshinodai, Chuo-ku, Sagamihara, Kanagawa 252-5210, Japan

${ }^{3}$ Department of Mathematical and Design Engineering, Faculty of Engineering, Gifu University

1-1 Yanagido, Gifu City 501-1193, Japan

${ }^{4}$ Department of Physics, Faculty of Science, Kagoshima University

1-21-24, Korimoto, Kagoshima 890-8580, Japan

5 INAF Istituto di Radioastronomia

via Gobetti 101, 40129 Bologna Italy

We observed NGC 4261 by multi-frequency phase-referencing method. Our core shift measurement determined the position of black hole to be $82 \pm 16 \mu$ as, which corresponds to $300 \pm 60$ $R_{\mathrm{s}}$, and $R_{\mathrm{S}}$ is the Schwarzschild radius, from $43 \mathrm{GHz}$ core. We also obtained spectral index maps by overlaying different frequency images accurately. Only a simple SSA model or spherical FFA model cannot describe our results of the core shift and spectral index map unrealistic. A possible solution is an additional component such as a thin disk in the limited region within $0.3 \mathrm{pc}$ on the counter side.

11th European VLBI Network Symposium \& Users Meeting,

October 9-12, 2012

Bordeaux, France

\footnotetext{
${ }^{*}$ Speaker.

†E-mail:haga@vsop.isas.jaxa.jp
} 


\section{Introduction}

Active galactic nuclei (AGNs) generate a huge amount of energy from very compact region within sub-parsecs. It is believed that the central engine of AGNs is composed of a super massive black hole (SMBH), accretion disk, and relativistic jets. These are most essential components for understanding the phenomena of AGNs.

NGC 4261 is one of the best candidates to investigate the spatial structure of circumnuclear disk and/or torus in sub-parsec scale by multi-frequency images. This source is a nearby FR-I type radio galaxy located at a distance of $31.6 \mathrm{Mpc}$ [1]. Hubble Space Telescope (HST) observations [2] revealed a $100 \mathrm{pc}$ scale disk of gas and dust and estimated the black hole mass kinematically to be $(4.9 \pm 1.0) \times 10^{8} M_{\odot}[3$. Previous VLBI observations showed that NGC 4261 had prominent two-sided jets to parsec scale inclined by $63^{\circ}$ from the line of sight [4]. A discontinuity in the intensity distribution of VLBI images is apparent in the counter jet. This region called as "gap" was interpreted as the obscuration by an edge-on, geometrically-thin, cold disk with the temperature of $\sim 10^{4} \mathrm{~K}[5]$.

There remains two problems. One is that the spectral index map in previous VLBI studies may not be accurate due to the core shift, because the positions of radio core is affected by synchrotron self absorption (SSA) and/or free-free absorption (FFA). Therefore, we can not use the core position directly as the reference position between different frequencies. Another problem is that the position of the black hole is unknown, which is the dynamical center of the putative accretion disk. Hada et al. (2011) 6] accurately determined the location of black hole in M 87 by multifrequency phase referencing observation. In this paper, we determined the black hole position by using the core shift and studied the structure of the absorber on the basis of the spectral index map that are free of the influence of the core shift.

\section{Observations}

Multifrequency phase-referencing observations of NGC 4261 and J1222+0413 were conducted using the Very Long Baseline Array (VLBA) at seven frequencies, from 1.4 to $43 \mathrm{GHz}$ quasisimultaneously. J1222+0413 is separated by $1.7^{\circ}$ from NGC 4261 and served as the reference position at each frequency.

\section{Results}

\subsection{Core shift measurement}

The core shift in the phase-referenced images of NGC 4261 appeared northwestward with respect to the core positions of $\mathrm{J} 1222+0413$. It is presumably the result of the combination of core shift originating in NGC 4261 and J1222+0413. The jet directions are almost perfectly perpendicular each other (east-west and north-south, respectively); therefore we can measure the core shifts of both sources. Fig.1(left) shows images of NGC 4261 at all frequencies, whose map centers are aligned with the $43 \mathrm{GHz}$ core. We fitted the core position of the approaching jet by least-square method with a power-law function of frequencies, $r(v)=\Omega \cdot v^{k}+c$, where $\Omega, v, k$ and $c$ are the scaling factor, frequency, the index parameter and the asymptotic value at infinity of frequency 
that indicates the starting point of a jet, respectively. From our result, the asymptotic parameter was determined within a tolerance of $32 \mu$ as and separated by $82 \mu$ as from $43 \mathrm{GHz}$ core position, which corresponds to $300 \pm 60 R_{\mathrm{s}}$. Moreover, we also measured the core positions in counter jet side of NGC 4261.

\subsection{Spectral index}

Spectral index ( $\alpha, S \propto v^{\alpha} ; S$ is a flux density) maps were produced from final cleaned images of two adjacent frequency using the AIPS task COMB at every frequency pair. In order to make accurate spectral index maps, the images between different frequencies were aligned on the basis of phase reference astrometry and we used the same beam equivalent to synthesized beam at the lower frequency .

Fig.1(right) shows the spectral index maps between 1-2 GHz pair and 5-8 GHz. The region of $\alpha>+2.5$ in these maps besides the $8-15 \mathrm{GHz}$ pair, suggesting FFA due to a circumnuclear absorber. This result is consistent with that by Jones et al. (2001) [5] that proposed highly-inclined thin disk as the free-free absorber intervening the counter jet.

\section{Discussion}

Hada et al. (2011) 6 determined the parameter c, which is interpreted as the location of the central engine of M 87, within 14 Rs from $43 \mathrm{GHz}$ core. On the other hand, Marscher et al. (2008)[7] pointed out the core was a standing shock point and then, the $43 \mathrm{GHz}$ core was far (about $10^{5} R_{\mathrm{s}}$ ) from the position of black hole in the BL Lac case. It is not obvious that the positions of black hole coincides with the asymptotic position at frequency infinity. On the other hand, our studies include the measurement of core shift on approaching jet and counter jet; we have successfully constrained the position of black hole between them (Fig.2)

We consider two possibilities of physical process to explain the core shift, which are SSA and FFA. Assuming a simple model with symmetry relative to the black hole, the index $k$ and the position of black hole $c$ common for the approaching side and counter jet side, the scaling factor $\Omega$ is examined. The differences in $\Omega$ between the two sides reflect the difference of the path length in the FFA case or beaming effect in the SSA case. The purple and blue curves in Fig. 2 show the expected profiles in the spherical FFA and simple SSA cases, respectively, on the counter side. As a result, a simple model solely cannot describe all the frequency points. A possible solution is the SSA core shift in addition to an absorbed component like a disk in limited region within 2 mas (corresponding to $0.3 \mathrm{pc}$ ) on the counter side.

\section{Acknowledgement}

We would like to thank "Radionet 3" for financial supports to attend this meeting and present a paper.

\section{References}

[1] Tonry, J. L., Dressler, A., Blakeslee, J. P., et al. 2001, ApJ, 546, 681 
[2] Jaffe, W., Ford, H. C., Ferrarese, L., van den Bosch, F., \& O’Connell, R. W. 1993, Nature, 364, 213

[3] Ferrarese, L., Ford, H. C., \& Jaffe, W. 1996, ApJ, 470, 444

[4] Piner, B. G., Jones, D. L., \& Wehrle, A. E. 2001, AJ, 122, 2954

[5] Jones, D. L., Wehrle, A. E., Piner, B. G., \& Meier, D. L. 2001, ApJ, 553, 968

[6] Hada, K., Doi, A., Kino, M., et al. 2011, Nature, 477, 185

[7] Marscher, A. P., Jorstad, S. G., D’Arcangelo, F. D., et al. 2008, Nature, 452, 966 


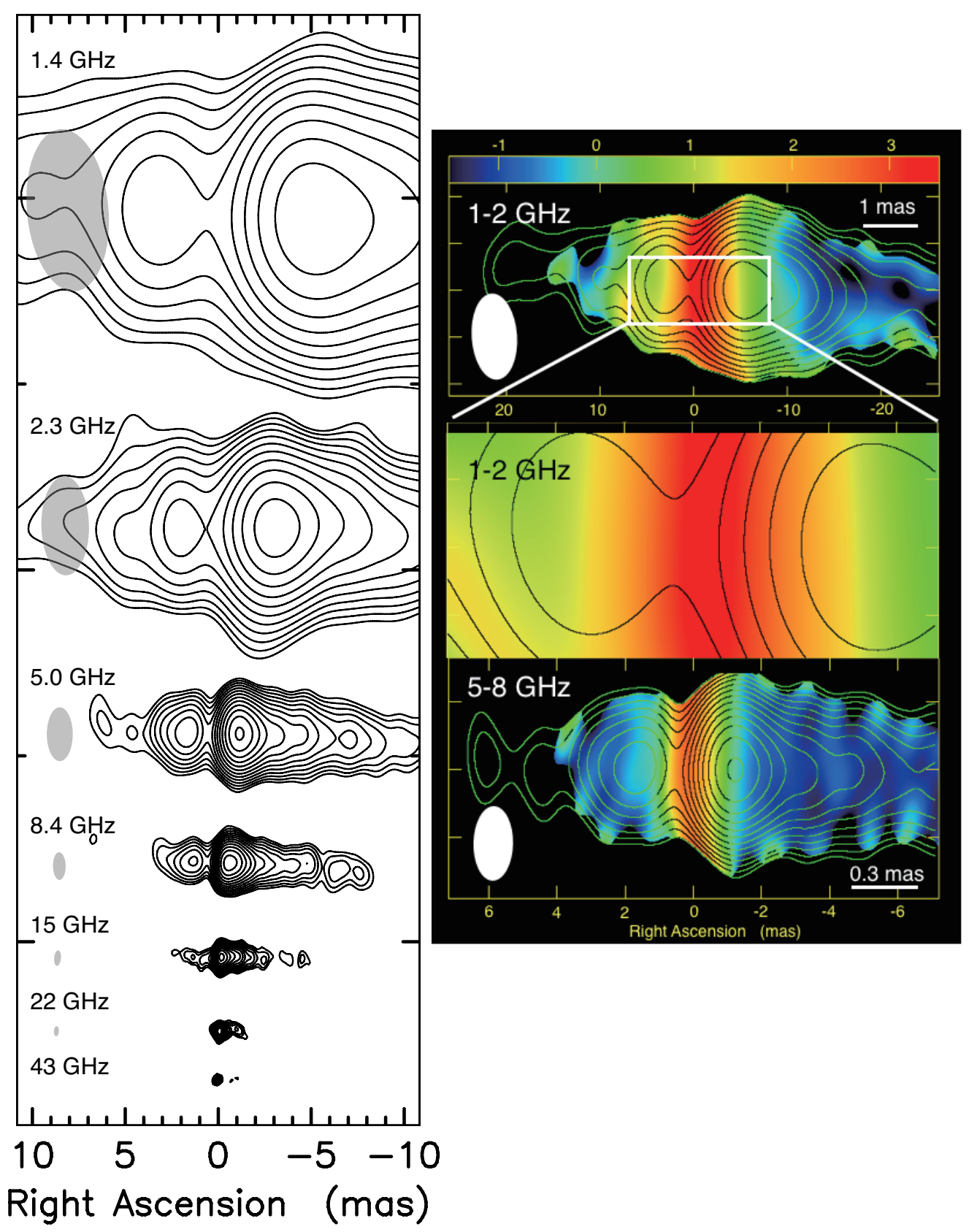

Figure 1: (Left) The self-calibrated images of NGC 4261 at 1.4-43 GHz, whose map centers are aligned relative to $43 \mathrm{GHz}$ core on the basis of phase-referencing astrometry. The gray ellipses are FWHMs of synthesized beam size. Contours start at $3 \sigma$ level of each frequency, increasing by factors of $\sqrt{2}$, where $\sigma$ is the image rms noise. (Right) The spectral index maps between $1 \mathrm{GHz}$ and $2 \mathrm{GHz}, 5 \mathrm{GHz}$ and $8 \mathrm{GHz}$ made after we restore the images with the lower frequency beams. 




Figure 2: Red and blue marks are the core positions of the approaching jet and the counter jet, respectively, in NGC 4261. The green line represents the fitted function by using least-square method for the approaching jet.The light blue and purple lines represent the expected core shifts of simple SSA case and spherical FFA case, respectively, which were calculated from $\Omega$ of approaching jet assuming that the $k$ and $c$ are common among both side. The positions of 5 and $8 \mathrm{GHz}$ deviate from the simple SSA. On the other hand, the spherical FFA case also does not describe all the frequencies. 